\title{
On a backward bifurcation of an epidemic model with capacities of treatment and vaccination
}

\author{
Hiromu Gion ${ }^{1}$, Yasuhisa Saito ${ }^{2 *}$ and Shigetoshi Yazaki ${ }^{3}$ \\ ${ }^{1}$ Artner Co.,Ltd., Sumitomo Nakanoshima Building 2F, 3-2-18 Nakanoshima, Kita-ku, Osaka \\ 530-0005, Japan \\ ${ }^{2}$ Department of Mathematics, Shimane University, 1060 Nishikawatsu-cho, Matsue-shi, Shi- \\ mane 690-8504, Japan \\ ${ }^{3}$ School of Science and Technology, Meiji University, 1-1-1 Higashi-Mita, Tama-ku, Kawasaki- \\ shi, Kanagawa 214-8571, Japan \\ *Corresponding author: ysaito@riko.shimane-u.ac.jp
}

Received April 05, 2021, Accepted June 12, 2021

\begin{abstract}
This paper presents an epidemic model with capacities of treatment and vaccination to discuss their effect on the disease spread. It is numerically shown that a backward bifurcation occurs in the basic reproduction number $\mathscr{R}_{0}$, where a stable endemic equilibrium co-exists with a stable disease-free equilibrium when $\mathscr{R}_{0}<1$, if the capacities are relatively small. This epidemiological implication is that, when there is not enough capacity for treatment or vaccination, the requirement $\mathscr{R}_{0}<1$ is not sufficient for effective disease control and disease outbreak may happen to a high endemic level even though $\mathscr{R}_{0}<1$.
\end{abstract}

Keywords epidemic, bifurcation, treatment, vaccine

Research Activity Group Mathematical Aspects on Continuum Mechanics

\section{Introduction}

Treatment is an important method to decrease the spread of diseases such as measles, tuberculosis and flu (see, for example [1]). In classical disease transmission models, the treatment rate of infective individuals is assumed to be proportional to their number. However, it is natural to think that there is some capacity for the treatment, including limited beds in hospitals, or an insufficient supply of medicine. Such a limited capacity, for example in [2], was considered into simple SIR ordinary differential equations (where the whole population is split up into susceptible, infective, and recovered individuals) by further assuming that the treatment rate keeps the same value when the number of infective individuals exceeds a certain number. This work has been recently modified by [3] and [4] to a model in a more realistic way such that infective individuals are divided into severe ones who need treatment and the others, as follows:

$$
\begin{aligned}
\frac{d S}{d t} & =A-\sigma_{m} S I_{m}-\sigma_{s} S\left[I_{s}\right]_{C_{I}}^{+}-\mu S \\
\frac{d I_{m}}{d t} & =\sigma_{m} S I_{m}+\sigma_{s} S\left[I_{s}\right]_{C_{I}}^{+}-(\mu+\rho+\beta) I_{m} \\
\frac{d I_{s}}{d t} & =\beta I_{m}-T\left(I_{s}\right)-\mu I_{s} \\
\frac{d R}{d t} & =T\left(I_{s}\right)+\rho I_{m}-\mu R
\end{aligned}
$$

where $S(t)$ and $R(t)$ denote the numbers of susceptible and recovered individuals at time $t$, respectively, while $I_{s}(t)$ and $I_{m}(t)$ denote the number of severe infective individuals who need treatment, and the number of nonsevere (that is, mild) infective individuals who do not need it. $A$ is the recruitment rate of the population, $\mu$ the per capita natural death rate of the population, $\rho$ the per capita natural recovery rate of infective individuals, $\sigma_{m}$ and $\sigma_{s}$ the disease transmission coefficients of mild and severe infective individuals, respectively, $\beta$ the severity coefficient of mild infective individuals. Furthermore, the treatment rate is defined as

$$
T\left(I_{s}\right)= \begin{cases}r I_{s}, & I_{s}<C_{I}, \\ r C_{I}, & I_{s} \geq C_{I}\end{cases}
$$

with the per capita treatment rate $r$ and the capacity $C_{I}$, and $\left[I_{s}\right]_{C_{I}}^{+}$is as

$$
\left[I_{s}\right]_{C_{I}}^{+}=\max \left\{0, I_{s}-C_{I}\right\}
$$

by which $\sigma_{s} S\left[I_{s}\right]_{C_{I}}^{+}$means the transmission rate of severe infective individuals exceeding the capacity. All the parameters are assumed to be positive constants.

Fig. 1 illustrates a solution behavior to the model (1) with the initial values

$$
S(0)=500, I_{m}(0)=60, I_{s}(0)=110, R(0)=0
$$

and the following parameters

$$
\begin{aligned}
& A=150, \quad \sigma_{m}=\sigma_{s}=0.001, \\
& \mu=0.1, \quad \rho=1, \quad \beta=0.5, \\
& r=0.8, \quad C_{I}=20 .
\end{aligned}
$$

Here and hereafter, we use the classical fourth-order Runge-Kutta method for numerical computation. Un- 


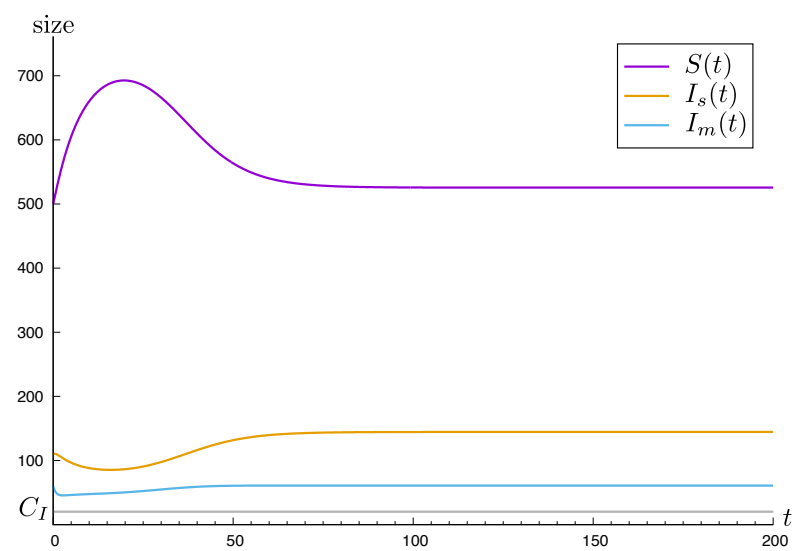

Fig. 1. Dynamics of a solution to the model (1) with the initial values (2) and the parameters (3). Trajectories $S(t), I_{s}(t), I_{m}(t)$, and the value of $C_{I}$ are drawn in purple, orange, light blue, and gray.

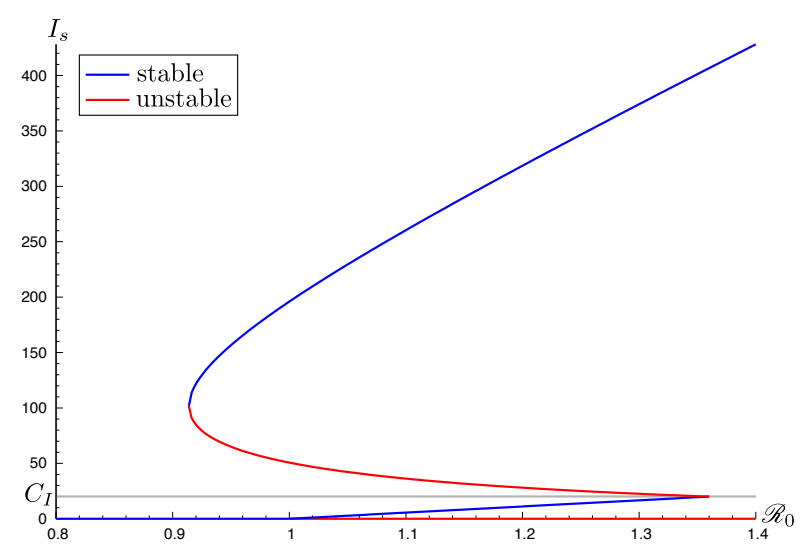

Fig. 2. Graph of the value $I_{s}$ in equilibrium versus $\mathscr{R}_{0}$ that shows a backward bifurcation diagram for the model (1) with parameters (3) (cf. [3]). The blue (resp. red) curve indicates stable (resp. unstable) equilibrium. The backward bifurcation starts from a point on the gray straight line (the value of $C_{I}$ ).

der these parameters and initial values, one can observe that both severe and mild infective individuals converge to positive constants, respectively.

The dynamics of disease transmission models is often characterized by the basic reproduction number $\mathscr{R}_{0}$, the average number of new cases that would be generated by a typical infected individual introduced into a completely susceptible population. In general, the phenomenon forward bifurcation is observed, where the disease-free equilibrium loses its stability and a stable endemic equilibrium appears as $\mathscr{R}_{0}$ increases through one. Both of [2] and [3] fully mathematically figured out backward bifurcations occur, where a stable endemic equilibrium co-exists with a stable disease-free equilibrium when $\mathscr{R}_{0}<1$ (as illustrated in Fig. 2), due to the low treatment capacity. The fact implies that the requirement $\mathscr{R}_{0}<1$ is not sufficient for effective disease control and disease outbreak can happen to a high endemic level even though $\mathscr{R}_{0}<1$. Indeed, under the parameters (3), we have $\mathscr{R}_{0}=0.9375$ and observe the convergence of infective individuals to a stable endemic equilibrium, as in Fig. 1.

As with treatment, vaccination is important to de-

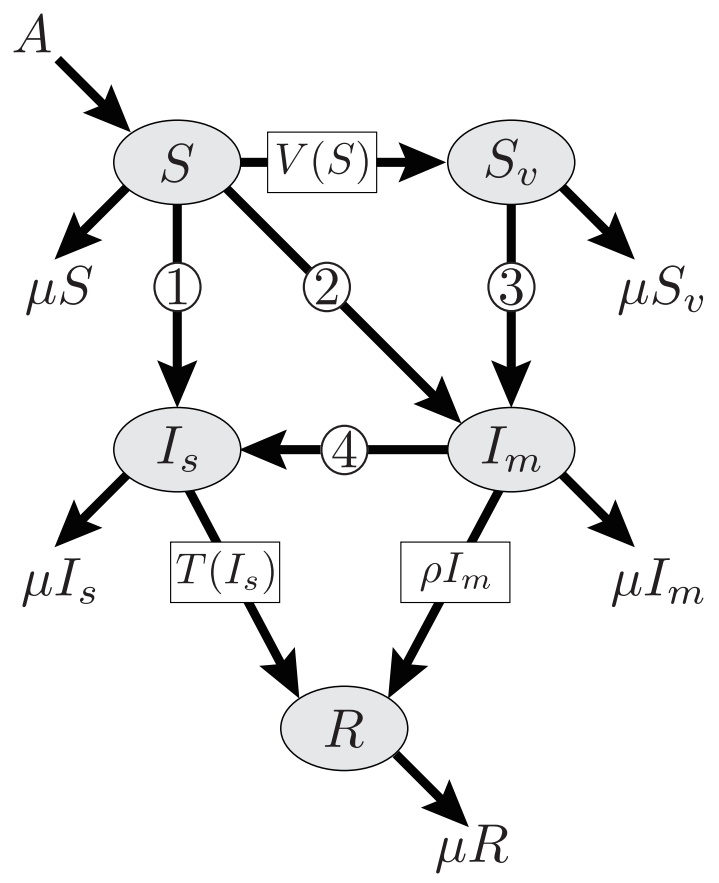

Fig. 3. Quantities of each circled number: (1) $\delta \sigma S\left(I_{m}+\left[I_{s}\right]_{C_{I}}^{+}\right)$, (2) $(1-\delta) \sigma S\left(I_{m}+\left[I_{s}\right]_{C_{I}}^{+}\right)$, (3) $\lambda S_{v}\left(I_{m}+\left[I_{s}\right]_{C_{I}}^{+}\right)$, (4) $\beta S I_{m} /(S+$ $\left.S_{v}\right)$.

crease the spread of diseases, and the number of vaccines is also practically limited. In this paper, based on [3], we consider an epidemic model with capacities of treatment and vaccination to discuss the effect of both these capacities on the disease spread. Our numerical simulations conclude that backward bifurcation probably occurs, which may lead to the same scenario as mentioned above even though vaccination works.

\section{A new epidemic model and the basic reproduction number}

Based on [3] and assuming a special case $\sigma_{s}=\sigma_{m}=\sigma$ for simplicity, we present a new epidemic model with capacities of treatment and vaccination. Fig. 3 depicts the conceptual diagram of our model and is realized as the following epidemic model:

$$
\begin{aligned}
\frac{d S}{d t}=A-\sigma S\left(I_{m}+\left[I_{s}\right]_{C_{I}}^{+}\right)-V(S)-\mu S, \\
\frac{d S_{v}}{d t}=V(S)-\lambda S_{v}\left(I_{m}+\left[I_{s}\right]_{C_{I}}^{+}\right)-\mu S_{v} \\
\frac{d I_{m}}{d t}=(1-\delta) \sigma S\left(I_{m}+\left[I_{s}\right]_{C_{I}}^{+}\right) \\
\quad+\lambda S_{v}\left(I_{m}+\left[I_{s}\right]_{C_{I}}^{+}\right)-\beta \frac{S I_{m}}{S+S_{v}} \\
\quad-(\mu+\rho) I_{m}, \\
\frac{d I_{s}}{d t}=\delta \sigma S\left(I_{m}+\left[I_{s}\right]_{C_{I}}^{+}\right)+\beta \frac{S I_{m}}{S+S_{v}}-T\left(I_{s}\right) \\
\quad-\mu I_{s}, \\
\frac{d R}{d t}=T\left(I_{s}\right)+\rho I_{m}-\mu R,
\end{aligned}
$$


where a new unknown function $S_{v}(t)$ denotes the number of vaccinated susceptible individuals at time $t$, and constant parameters $\lambda, \delta$ and a function $V$ are newly introduced. $\lambda(\lambda \geq 0)$ is the disease transmission coefficient of mild and severe infective individuals to vaccinated susceptible individuals, $\delta(0 \leq \delta \leq 1)$ the fraction that divides the infected individuals $\sigma S\left(I_{m}+\left[I_{s}\right]_{C_{I}}^{+}\right)$into severe ones and mild ones, $V(S)$ the vaccination rate defined as

$$
V(S)= \begin{cases}v S, & S<C_{V} \\ v C_{V}, & S \geq C_{V}\end{cases}
$$

with the per capita constant rate $v \geq 0$ and the constant capacity $C_{V}$. Also, the severity transition rate is assumed to be

$$
\beta \frac{S I_{m}}{S+S_{v}}
$$

in a simplest way since it should be negatively correlated with the vaccine spread. We give the initial data as

$$
\begin{aligned}
& S(0)>0, \quad S_{v}(0) \geq 0, \quad I_{m}(0) \geq 0, \quad I_{s}(0) \geq 0, \\
& I_{m}(0)+I_{s}(0)>0, \quad R(0) \geq 0 .
\end{aligned}
$$

Clearly, for the case $v=0, S_{v}(0)=0$, and $\delta=0$, (4) is reduced to (1) with $\sigma_{s}=\sigma_{m}=\sigma$.

Disease-free equilibrium is required to derive the basic reproduction number $\mathscr{R}_{0}$. According to the concept of next generation matrix, let

$$
x={ }^{t}\left(I_{m}, I_{s}, S, S_{v}\right) .
$$

Then (4) is written as

$$
\frac{d x}{d t}=\mathscr{F}(x)-\mathscr{V}(x)
$$

where $\mathscr{F}$ is the rate of production term of new infection and $-\mathscr{V}$ otherwise. From the viewpoint of local behavior in completely susceptible population, the first principal matrices (defined as $\mathcal{F}, \mathcal{V}$ below) of the Jacobian matrix of $\mathscr{F}$ and $\mathscr{V}$ at disease-free equilibrium play a key role of defining $\mathscr{R}_{0}$ (see $[5,6]$ ). For $(4)$, the disease-free equilibrium is expressed alternatively as

$$
\begin{aligned}
& \left(I_{m}, I_{s}, S, S_{v}\right) \\
& \quad= \begin{cases}\left(0,0, \frac{A}{\mu+v}, \frac{A v}{\mu(\mu+v)}\right) & \text { if } A \leq(\mu+v) C_{V}, \\
\left(0,0, \frac{A-v C_{V}}{\mu}, \frac{v C_{V}}{\mu}\right) & \text { if } A>(\mu+v) C_{V},\end{cases}
\end{aligned}
$$

and the first principal matrices $\mathcal{F}, \mathcal{V}$ are given as

$$
\begin{aligned}
& \mathcal{F}=\left(\begin{array}{cc}
\frac{A\{\mu(1-\delta) \sigma+v \lambda\}}{\mu(\mu+v)} & 0 \\
\frac{A \delta \sigma}{\mu+v} & 0
\end{array}\right), \\
& \mathcal{V}=\left(\begin{array}{cc}
\frac{\mu \beta+(\mu+v)(\mu+\rho)}{\mu+v} & 0 \\
-\frac{\mu \beta}{\mu+v} & \mu+r
\end{array}\right)
\end{aligned}
$$

if $A \leq(\mu+v) C_{V}$, while

$$
\begin{aligned}
& \mathcal{F}=\left(\begin{array}{cc}
\frac{A(1-\delta) \sigma-v\{(1-\delta) \sigma-\lambda\} C_{V}}{\mu} & 0 \\
\frac{A \delta \sigma-v \delta \sigma C_{V}}{\mu} & 0
\end{array}\right), \\
& \mathcal{V}=\left(\begin{array}{cc}
\frac{A(\mu+\beta+\rho)-v \beta C_{V}}{A} & 0 \\
\frac{-A \beta+v \beta C_{V}}{A} & \mu+r
\end{array}\right)
\end{aligned}
$$

if $A>(\mu+v) C_{V}$. Hence, the basic reproduction number for (4) is

$$
\mathscr{R}_{0}=\left\|\mathcal{F} \mathcal{V}^{-1}\right\|=\frac{A\{\mu(1-\delta) \sigma+v \lambda\}}{\mu\{\mu \beta+(d+v)(\mu+\rho)\}}
$$

if $A \leq(\mu+v) C_{V}$, while

$$
\begin{aligned}
\mathscr{R}_{0} & =\left\|\mathcal{F} \mathcal{V}^{-1}\right\| \\
& =\frac{A\left[A(1-\delta) \sigma-v\{(1-\delta) \sigma-\lambda\} C_{V}\right]}{A \mu(\mu+\beta+\rho)-v \mu \beta C_{V}}
\end{aligned}
$$

if $A>(\mu+v) C_{V}$, where $\|\mathcal{M}\|$ represents the spectral radius of the matrix $\mathcal{M}$.

\section{Numerical results: effect of the two ca- pacities and a backward bifurcation}

For (4), it follows from [6] that the disease-free equilibrium is asymptotically stable if $\mathscr{R}_{0}<1$, but unstable if $\mathscr{R}_{0}>1$. Without any backward bifurcation structures, the disease-free equilibrium is usually expected to attract all positive solutions of (4) when $\mathscr{R}_{0}<1$ (see Figs. 4 and 7). A number of numerical works, however, suggest that a backward bifurcation exists if capacities of treatment and vaccination are relatively small (see Figs. 5 and 6).

In order to show the vaccination effect, in addition to (3), we will use the parameters

$$
\sigma=0.001, \quad \lambda=0.9 \delta \sigma=0.00018<\sigma, \quad v=0.9,
$$

and $C_{V}=36$ or 37 . In the case where the vaccination capacity $C_{V}=37$, some vaccination effect is numerically observed where $I_{m}(t)$ and $I_{s}(t)$ converge to zero, shown in Fig. 4, even if we use the same parameters and initial values as in Fig. 1. Decreasing $C_{V}$ from 37 to 36, however, we observe that a solution does not converge to a disease-free equilibrium but to a stable endemic equilibrium, even if $\mathscr{R}_{0}<1$, shown in Fig. 5 .

These facts suggest that a backward bifurcation exists for (4) if the vaccination capacity is relatively small. Indeed, direct numerical works Fig. 6 show that a stable endemic equilibrium co-exists with a stable disease-free equilibrium when $\mathscr{R}_{0}<1$. This means that a backward bifurcation may exist in a similar situation as in Fig. 2. Furthermore, Fig. 7 shows that increasing $C_{I}$ leads to no backward bifurcation structures, where the disease-free equilibrium becomes asymptotically stable if $\mathscr{R}_{0}<1$, but unstable if $\mathscr{R}_{0}>1$. That is what we mentioned in the opening paragraph of this section. 


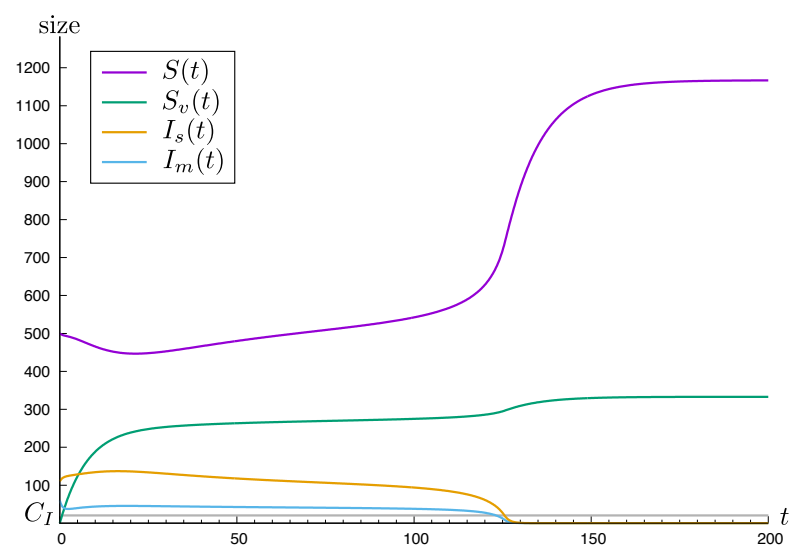

Fig. 4. Dynamics of a solution to the model (4) with the initial values (2) and $S_{v}(0)=0$, and with the parameters (3), (5), and $C_{V}=37$, where convergence to a disease-free equilibrium is observed. Trajectories $S(t), I_{s}(t), I_{m}(t)$, and the value of $C_{I}$ are drawn in purple, orange, light blue, and gray. We have $\mathscr{R}_{0}=$ 0.667253 .

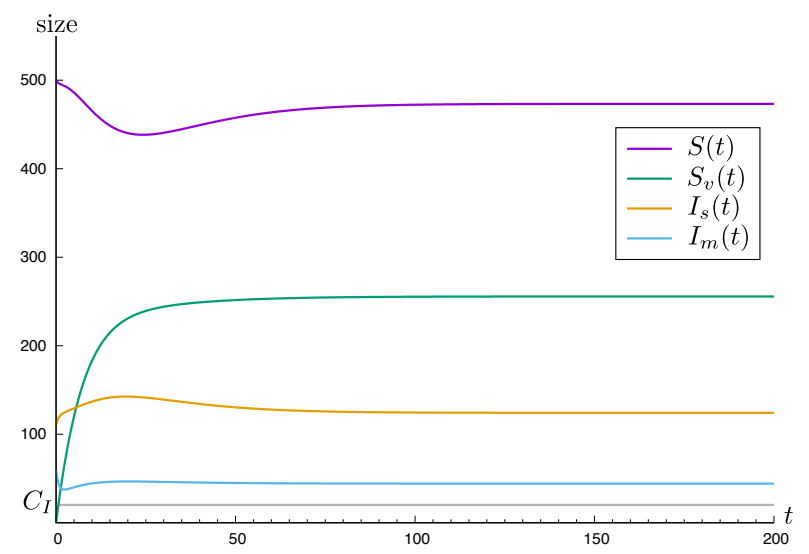

Fig. 5. Dynamics of a solution to the model (4) when $C_{V}=36$, where convergence to a stable endemic equilibrium is observed although $\mathscr{R}_{0}$ is less than $1\left(\mathscr{R}_{0}=0.669651\right)$. Other parameters, the initial values, and the colors of the curves are all the same as in Fig. 4.

\section{Concluding remarks}

Treatment and vaccination cannot be considered independently since they are practically done by a limited number of common medical professionals. This means that these capacities have a trade-off relationship. In other words, our proposed model is essentially realistic compared with a simple SIR model and its variations.

Based on numerical results in this paper, analysis should be developed to fully mathematically ensure the existence of backward bifurcation structure, and furthermore to clarify optimal capacity distribution for treatment and vaccination against the backward bifurcation scenario. We leave it for future work.

\section{Acknowledgments}

This work is partially supported by JSPS KAKENHI Grant Numbers 20K03750 (YS) and $19 \mathrm{H} 01807$ (SY). The authors would like to thank the referee for her or his variable comments.

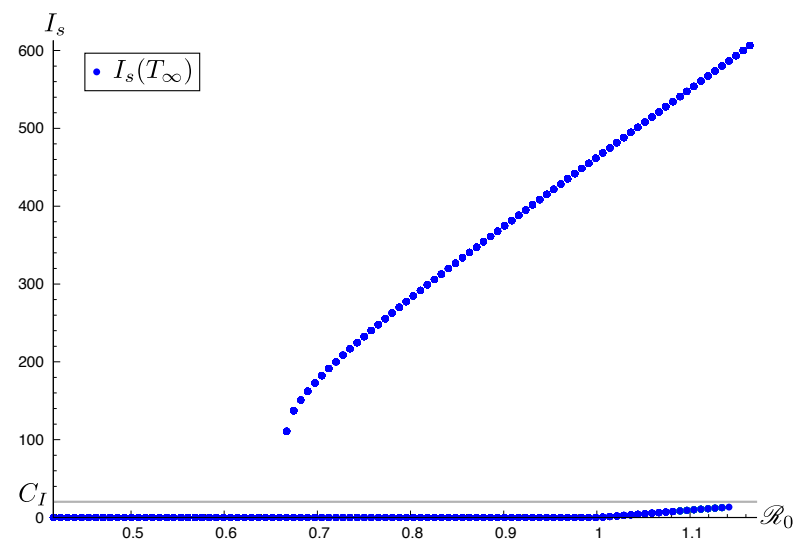

Fig. 6. Plots of a pair of $\left(\mathscr{R}_{0}, I_{s}\left(T_{\infty}\right)\right)$ at a large value $T_{\infty}=1000$ when $C_{V}=36$ and $C_{I}=20$. They are calculated with $101^{2}$ samples of $\left(A, I_{s}(0)\right) \in[100,250] \times[0,250]$, and $S_{v}(0)=0$, where other parameters and initial values are the same as in (3), (5), and (2), respectively.

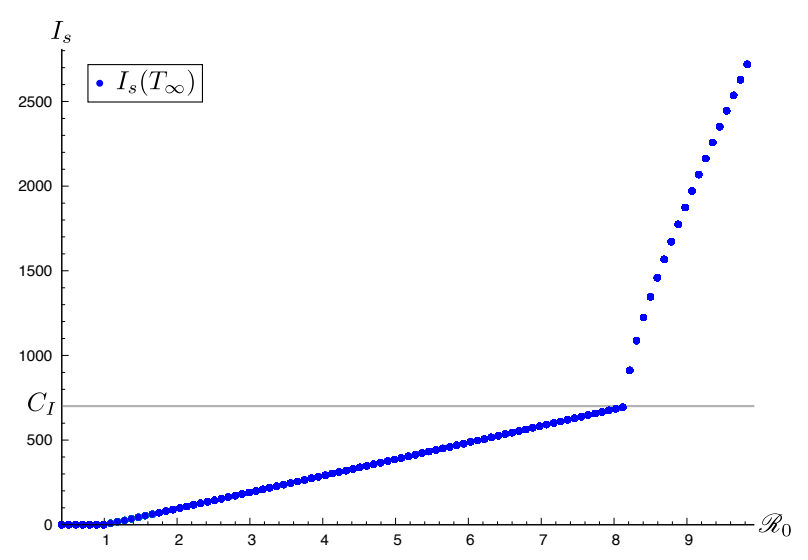

Fig. 7. Plots of a pair of $\left(\mathscr{R}_{0}, I_{s}\left(T_{\infty}\right)\right)$ at a large value $T_{\infty}=1000$ when $C_{V}=36$ and $C_{I}=700$. They are calculated with $101^{2}$ samples of $\left(A, I_{s}(0)\right) \in[100,2000] \times[0,250]$, and $S_{v}(0)=0$, where other parameters and initial values are the same as in (3), (5), and (2), respectively.

\section{References}

[1] H. R. Thieme, Mathematics in Population Biology, Princeton University Press, Princeton, 2003.

[2] W. Wang, Backward bifurcation of an epidemic model with treatment, Math. Biosci., 201 (2006), 58-71.

[3] H. Gion and Y. Saito, Backward bifurcation of a diseaseseverity-structured epidemic model with treatment, arXiv:2110.01505 [q-bio.PE].

[4] Y. Saito, Backward bifurcation caused by treatment capacity in a disease transmission model (in Japanese), Bull. JSIAM, 31 (2021), 13-21.

[5] O. Diekmann, J. A. P. Heesterbeek and J. A. J. Metz, On the definition and the computation of the basic reproduction ratio $R_{0}$ in models for infectious diseases in heterogeneous populations, J. Math. Biol., 28 (1990), 365-382.

[6] P. van den Driessche and J. Watmough, Reproduction numbers and sub-threshold endemic equilibria for compartmental models of disease transmission, Math. Biosci., 180 (2002), 29-48. 